Pacific Journal of Mathematic 


\title{
THE INDEX OF A GROUP IN A SEMIGROUP
}

\author{
GeORge M. BergmaN
}

\begin{abstract}
We shall define the right and left indices of a subgroup $G$ in a semigroup $S$ with unit, and show that if $G$ has cancellation in $S$, and at least one of these indices is finite, then they are equal. If only right cancellation holds, and the left index is finite, the right index will be either less than the left index, or infinite. It will be shown by counterexamples that these theorems are "best results."
\end{abstract}

Let $S$ be a semigroup with unit and $G$ a subgroup of $S$ with the same unit. Let $[S: G]_{\text {, }}$ denote the cardinality of the set of right cosets $x G$ of $G$ in $S$, and $[S: G]_{\ell}$ the cardinality of the set of left cosets. These are equal when $S$ is a group, because the operation $x \mapsto x^{-1}$ gives an anti-automorphism of $S$ and of $G$.

For $S$ any finite semigroup, we see by a counting argument that these two indices will be equal if $G$ has left and right cancellation in $S$ :

$$
\begin{array}{ll}
g x=x \Rightarrow g=e & (x \in S, g \in G), \\
x g=x \Rightarrow g=e & (x \in S, g \in G) .
\end{array}
$$

If only one of the cancellation conditions is assumed, say (2), then we see $[S: G]_{\imath} \leqq[S: G]_{\ell}$. As indicated above, we shall generalize these results, replacing the assumption that $S$ is finite by assumptions that one or both of the indices is. But let us begin with two counterexamples, one showing that one-sided cancellation is not enough to imply equal indices even for finite $S$; the other, that assuming cancellation, the two indices can still be any two infinite cardinals.

\section{Counterexamples. (a) Assuming only right cancellation.}

Let $X$ be a set, $G$ a simply transitive group of permutations of $X$, and $S$ the semigroup of maps of $X$ into itself consisting of the elements of $G$ and the constant maps, all written on the right. Then it is easy to see that $G$ has right cancellation in $S$, and that $[S: G]_{2}=2$, $G$ forming one coset, and the constant maps another; but $[S: G]_{\ell}=$ $1+|X|$ : each constant map comprises a left coset. In this example, if $X$ is finite, $S$ will also be.

(b) With cancellation, but infinite indices. Let $G$ be a group with a one-to-one endomorphism $\alpha$ such that $[G: \alpha(G)]>\aleph_{0}$. (E.g., let $H$ be an uncountable group, $G=H \oplus H \oplus H \oplus \cdots$, and $\alpha$ the "right shift" endomorphism.) Let $S$ be the semigroup of all expressions $x^{n} g(n \geqq 0, g \in G)$, with multiplication defined using the law $g x^{n}=$ 
$x^{n} \alpha^{n}(g)$. Then it is easy to verify that $S$ is a semigroup with cancellation (in fact, embeddable in a group), and that elements $x^{n} g$ and $x^{n} h$, though they always belong to the same right $G$-coset, will belong to the same left coset if and only if $g h^{-1} \in \alpha^{n}(G)$. Hence $[S: G]_{2}=$ $1+1+\cdots=\boldsymbol{\aleph}_{0}$, but $[S: G]_{\iota}=1+[G: \alpha(G)]+\left[G: \alpha^{2}(G)\right]+\cdots=$ $[G: \alpha(G)]>\boldsymbol{\aleph}_{0}$. By using more indeterminates $x$, all acting in the same way on $G$, one can get arbitrary cardinals as left and right indices.

2. In the next five sections, we shall prove the two theorems mentioned in the introduction.

Let us begin with some general observations on a semigroup $S$ with unit, having a subgroup $G$ with $[S: G]_{\iota}<\infty$.

Given any $x \in S$, define $G_{x} \subseteq G$ as $\{g \in G \mid x g \in G x\}$. This will be a subgroup of $G$ : in fact, it is the isotropy subgroup of the coset $G x$ under the action of $G$, by right multiplication, on the class of left cosets of $G$ in $S$. Since this class is finite, $\left[G: G_{x}\right]<\infty$.

If $G$ satisfies left and right cancellation in $S$, the correspondence between elements $g$ and $h$ satisfying $x g=h x$ for given $x$ is one-to-one and will constitute a monomorphism of groups, $\varphi_{x}: G_{x} \rightarrow G$; what we shall call, in the next section, a "partial monomorphism" of $G$ into itself.

Dropping cancellation again, we note that if $x$ is any element of $S$, the family of cosets $G x, G x^{2}, G x^{3}, \ldots$ will be finite, hence we can find positive integers $a, b$ such that $G x^{a}=G x^{a+b}$. Hence $G x^{a}=G x^{a+m b}$ for all $m \geqq 1$. Taking $m=a$ and multiplying on the right by $x^{a(b-1)}$, we get $G x^{a b}=G x^{2 a b}$, in other words, there is some positive power $y$ of $x$ such that $G y=G y^{2}$, and we can write $y^{2}=g y$. It follows that for any $n \geqq 0, y^{n+1}=g^{n} y$.

Now again assume right and left cancellation of $G$ in $S$. Since $G_{y}$ is of finite index in $G$, there will exist $c>0$ such that $g^{c} \in G_{y}$. We calculate:

$$
g^{c+1} y=y^{c+2}=y g^{c} y=\varphi_{y}\left(g^{c}\right) y y=\varphi_{y}\left(g^{c}\right) g y .
$$

Using left cancellation, we conclude that $\varphi_{y}\left(g^{c}\right)=g^{c}$, i.e., $y g^{c}=g^{c} y$. It follows that $y^{2 c}=g^{c} y^{c}=y^{c} g^{c}$. Putting $z=y^{c}, h=g^{c}$, we get an equation $z^{2}=z h$, where $z$ is again a power of $x$.

3. Partial monomorphisms. By a partial monomorphism $f$ from a group $G$ to a group $H$, we shall mean a monomorphism of groups, from a subgroup $G_{f}$ of finite index in $G$, into the group $H$. We shall call two partial monomorphisms $f, g: G \rightarrow H$ equivalent if they agree on some subgroup of finite index in $G$ (not necessarily all of $G_{f} \cap G_{g}$ ). 
This is an equivalence relation. (However we shall not here identify equivalent partial monomorphisms.)

By the composition of two partial monomorphisms, $F \stackrel{f}{\longrightarrow} G \stackrel{g}{\longrightarrow} H$, we shall mean the partial monomorphism $g f$ defined on $F_{g f}=f^{-1}\left(G_{g}\right) \leqq F_{f}$. It is easy to show that this sort of composition respects equivalence.

(Thus one may form a category whose objects are groups, and whose morphisms are equivalence classes of partial monomorphisms, or more generally, of partial homomorphisms, analogously defined. This category might be worthy of some study.)

Note that the images of two equivalent partial homomorphisms $f, g: F \rightarrow G$ will be commensurable subgroups of $G$, that is, they have a common subgroup which is of finite index in each of them.

Define the index of a partial monomorphism $f: G \rightarrow H$ as the quotient $\left.\nu_{f}=\left[G: G_{f}\right] /\left[H: f, G_{f}\right)\right]$, or zero if the denominator of this expression is infinite-in either case, a nonnegative rational number. It is easy to check that the indices of equivalent partial monomorphisms are equal (it is in order that this be true that we have restricted our attention to monomorphisms, rather than defining partial homomorphisms), and with the help of this fact, one checks that the index of a composition of partial monomorphisms is the product of their indices.

Note that for partial monomorphisms, $F \stackrel{f}{\longrightarrow} G \stackrel{g}{\longrightarrow} H$, the image of $g f$ will have finite index in the image of $g$ if and only if $\nu_{f} \neq 0$.

4. Back to semigroups. Once more let $S$ be a semigroup with unit, and $G$ a subgroup with the same unit, having two-sided cancellation in $S$, and such that $[S: G]_{c}$ is finite.

The maps $\varphi_{x}: G_{x} \rightarrow G$ defined in $\S 2\left(\varphi_{x}(g)=h\right.$ if $\left.x g=h x\right)$ will be partial monomorphisms of $G$ into itself. Note that we are writing $G_{x}$ where the terminology of $\S 3$ would have $G_{\varphi_{x}}$. We shall similarly put $\nu_{x}$ for $\nu_{\varphi_{x}}$.

Given $x, y \in S$, we see that $\varphi_{x y}$ is an extension of $\varphi_{x} \varphi_{y}$, so in particular, these are equivalent partial monomorphisms. Hence $\nu$ will be a homomorphism of semigroups from $S$ into the multiplicative semigroup of nonnegative rationals. Note that $\nu_{g}=1$ for $g \in G$.

Suppose $\nu_{x}=0$ for some $x \in S$. By the argument given in $\S 2$, some power $z$ of $x$ will satisfy $z^{2}=z h$ for some $h \in G$. Now the image of $\varphi_{z} \varphi_{z}$ will have infinite index in the image of $\varphi_{z}$, because $\nu_{z}=0$, but it will also be commensurable with the image of the equivalent partial monomorphism $\varphi_{z^{2}}=\varphi_{z h}$, which is in turn commensurable with the image of $\varphi_{z} \varphi_{h}$, which has finite index in the image of $\varphi_{z}-$-contradiction!

Hence $\nu$ will be a multiplicative homomorphism of $S$ into the positive rationals. But since it sends $G$ to $\{1\}$, and $G$ has finite left 
index in $S$, its image will be a finite subsemigroup of the positive rationals-and the only such semigroup is $\{1\}$. Hence $\nu_{x}=1$ for all $x \in S$, i.e., $\left[G: \varphi_{x}\left(G_{x}\right)\right]=\left[G: G_{x}\right]$.

5. Measure for measure. Let us define two finitely additive measures $\mu_{c}$ and $\mu_{z}$ on the set $S$, taking for a basis of measurable sets of $\mu_{l}$ the left cosets $H x$ of subgroups $H$ of finite index in $G$, to each of which we assign the measure $1 /[G: H]$, and defining the second measure similarly in terms of right cosets. It is clear that these are consistent definitions, and $\mu_{\ell}(S)=[S: G]_{\ell}$, while $\mu_{z}(S)$ is defined if and only if $[S: G]_{2}$ is finite, and if this is so, equals $[S: G]_{2}$.

Given $x \in S$ let us define the semicoset of $x$ as $G x \cap x G=x G_{x}=$ $\varphi_{x}\left(G_{x}\right) x$. Now left translation by the elements of $G$ transitively permutes the semicosets which form a given left coset $G x$, and we see that the number of them will be $\left[G: \varphi_{x}\left(G_{x}\right)\right]$. Because $\nu_{x}=1$, this equals $\left[G: G_{x}\right]$, which we know is finite. Since there are only finitely many left cosets $G x$, the number of semicosets will be finite.

The semicosets $x G_{x}=\varphi_{x}\left(G_{x}\right) x$ will be measurable under $\mu_{z}$ with measure $1 /\left[G: G_{x}\right]$, and under $\mu_{c}$ with measure $1 /\left[G: \varphi_{x}\left(G_{x}\right)\right]$. As we just observed, these numbers are equal. Hence we have partitioned $S$ into finitely many $\mu_{\varkappa}$-and- $\mu_{\ell}$ measurable sets on each of which these two measures have the same value. So $\mu_{\ell}(S)=\mu_{2}(S)$, completing the proof of:

THEOREM 1. Let $S$ be a semigroup with unit $e$, and $G \subseteq S$ a subgroup with the same unit, having left and right cancellation in S. Then if $[S: G]_{\ell}<\infty$, we have $[S: G]_{\iota}=[S: G]_{\ell}$.

By symmetry, the same is true if $[S: G]_{2}<\infty$.

6. One-sided cancellation. Now let us suppose only that $G$ has right cancellation in $S$, and $[S: G]_{\ell}<\infty$.

The subgroups $G_{x}$ defined in $\S 2$ still have finite index in $G$. Since $G_{x}$ depends only on the coset $G x$, there are only finitely many distinct groups $G_{x}$, hence their intersection, which we shall call $G_{0}$, also has finite index in $G$.

Let us measure the failure of the left cancellation law by writing, for each $x \in S, H_{x}=\{g \in G \mid g x=x\}$, the isotropy subgroup of $x$. We can now prove:

THEOREM 2. Let $S$ be a semigroup with unit $e$, and $G \leqq S$ a subgroup with the same unit, satisfying right cancellation: $x g=x \Rightarrow$ $g=e(x \in S, g \in G)$, and having finite left index: $[S: G]_{\ell}<\infty$. Then either of the hypotheses: 
(a) $[S: G]_{2}<\infty$

(b) None of the isotropy subgroups $H_{x} \subseteq G$ is conjugate in $G$ to a proper subgroup of itself,

implies:

(c) $[S: G]_{2} \leqq[S: G]_{\ell}$,

(d) the subgroup $G_{0}=\{g \in G \mid \forall x \in S, x g \in G x\}$ (of finite index in $G$ ) has left (as well as right) cancellation in $S$,

(e) Each isotropy subgroup $H_{x} \subseteq G$ has only finitely many distict conjugates, as a subgroup of $G$,

(f) There are only finitely many distinct isotropy subgroups $H_{x}$,

(g) Every isotropy subgroup $H_{x} \subseteq G$ is finite.

In fact, conditions (a)-(g) are all equivalent.

Proof. The scheme of proof will be:

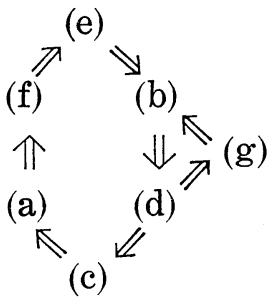

Aside from an application of Theorem 1, the one key step, with which we shall begin, is:

(b) $\Rightarrow(d)$ : We wish to prove that for all $x \in S, H_{x} \cap G_{0}=\{e\}$. Suppose we have an $x$ for which this is not true. By the argument given in $\S 2$ we can find a positive power $y$ of $x$ such that $y^{2}=g y$ for some $y \in G$. Note that $H_{y^{2}} \supseteqq H_{y} \supseteqq H_{x}$. Let $h$ be an element of $H_{y} \cap G_{0}-\{e\}$. Since $h \in G_{0} \subseteq G_{y}$, we can write $y h=i y(i \in G)$. By right cancellation we have $y \neq y h=i y$, so $i \notin H_{y}$. But $i y^{2}=y h y=y^{2}$, so $i \in H_{y^{2}}=H_{g y}=g\left(H_{y}\right) g^{-1}$. Hence $H_{y^{2}}$ is conjugate to a proper subgroup, $H_{y}$, of itself, contradicting (b).

$(d) \Rightarrow(\mathrm{g})$ : By $(\mathrm{d})$, no $H_{x}$ meets the subgroup $G_{0}$, hence each $H_{x}$ must have order $\leqq\left[G: G_{0}\right]$.

$(\mathrm{g}) \Rightarrow(\mathrm{b})$ : Immediate.

$(d) \Rightarrow$ (c): $\quad G_{0}$ will have finite left index in $S$, so we can apply Theorem 1 to conclude $\left[S: G_{0}\right]_{\iota}=\left[S: G_{0}\right]_{\iota}$. Comparing $G$-cosets and $G_{0}$-cosets, we get $[S: G]_{\iota}=\left[S: G_{0}\right]_{\iota} /\left[G: G_{0}\right]=\left[S: G_{0}\right]_{\ell} /\left[G: G_{0}\right] \leqq[S: G]_{\iota}$.

(c) $\Rightarrow$ (a): Immediate.

(a) $\Rightarrow$ (f): It is easy to see that two elements of the same right coset have the same left isotropy subgroup. This makes the implication clear.

(f) $\Longrightarrow(e)$ : For, any conjugate of an isotropy subgroup is an isotropy 
subgroup: $g H_{x} g^{-1}=H_{g x}$.

(e) $\Rightarrow$ (b): If $g H_{x} g^{-1} \subset H_{x}$, then clearly $H_{x} \subset g H_{x} g^{-1} \subset g^{2} H_{x} g^{-2} \subset \cdots$ are all distinct, contradicting (e).

One can state many special cases and corollaries to this result. For instance, if $G$ has right cancellation and finite right index in $S$, then $[S: G]_{\ell} \geqq[S: G]_{2}$. If $G$ is a group with ascending chain condition on subgroups, or an abelian group then condition (b) is automatic, hence given $S \supseteqq G$ as in Theorem 2 , all of (a)-(g) hold.

A question we have not been able to answer is: if $G$ in Theorem 2 is finitely generated, or more generally, if $S$ is finitely generated, must conditions (a)-(g) hold? (A finitely generated group can have a subgroup properly containing a conjugate of itself. E.g., the group defined by two generators $a$ and $b$, and one relation $a b a^{-1}=b^{2}$.)

7. Another counterexample. We shall now give a rather complicated example (perhaps necessarily so) showing that conditions (a)(g) of Theorem 2 can, in fact, fail to hold.

Let $G$ be a free group on countably many generators $g_{0}, g_{1}, \cdots$.

Let $S$ be obtained by adjoining to $G$, as a semigroup with unit, one generator $y$, and the relations

$$
y y=g_{0} y,
$$

$$
y g_{i}^{ \pm 1}=g_{i+1}^{ \pm 1} y, \quad(i=0,1, \cdots) .
$$

(Actually, the conditions (4) with exponent -1 are implied by those with +1.)

Applying (3) and (4) to the expression $y y g_{\imath-1}^{ \pm 1}(i=1,2, \cdots)$ in the two possible orders, we get the equations $g_{\imath+1}^{ \pm 1} g_{0} y=g_{0} g_{\imath}^{ \pm 1} y$. Applying (3) and (4) to yyy in the two possible ways, we get $g_{1} g_{0} y=g_{0} g_{0} y$, i.e., the $i=0$ case of the preceding equation with exponent +1 only. Multiplying on both sides by $g_{1}^{-1}$ and transposing, we get $g_{1}^{-1} g_{0}^{2} y=g_{0} y$.

Finally, we can take each of the above equations, multiply on the right by an arbitrary sequence $F_{0}$ of $g_{3}^{ \pm 1}$ 's and $y$ 's, and apply (3) and (4) to get:

$$
\left.\left\{\begin{array}{l}
g_{i+1}^{ \pm 1} g_{0} F y=g_{0} g_{i}^{ \pm 1} F y \\
\text { where } i=0,1, \cdots, \text { but only the exponent } \\
+1 \text { is allowed for } i=0 \text {, and } \\
g_{1}^{-1} g_{0}^{2} F y=g_{0} F y .
\end{array}\right\} \text { in } g_{0}, g_{1}^{ \pm 1}, g_{2}^{ \pm 1}, \cdots\right) .
$$

To have a set of relations defining $S$ as a semigroup, we also want to write down the semigroup-relations defining the free group $G$ : 


$$
\begin{aligned}
& g_{i} g_{i}^{-1}=e, \\
& g_{\imath}^{-1} g_{i}=e
\end{aligned} \quad(i=0,1, \cdots) .
$$

We now claim that the elements of $S$ can be uniquely represented by the set of words $w$ in the $g_{i}^{ \pm 1}$ and $y$ not involving any of the expressions on the left-hand side of equations (3)-(6). This means, in fact, words $w=A$, where $A$ is a reduced expression for an element of the free group $G$, and words $w=A y$, where $A$ is as before, and $w$ does not end in one of the left-hand expressions of (5).

It is clear on the one hand that repeated arbitrary applications of the reductions (3)-(6) will eventually bring any word to such a form. To show the uniqueness of this expression, it suffices to verify that when we have an overlap of two reducible expressions of the sort given by (3)-(6), the results of the two possible reductions can be further reduced to some common expression. (Cf. [1, Th. III. 9.3].)

All but one of these calculations are either trivial, or reduce to calculations made in the preceding paragraphs, and we leave the verification to the reader. The one case requiring special consideration is that of an expression $y A y$, where $A y$ is the left-hand side of one of the equations of (5). Let us denote by $B y$ the right-hand side of the same equation.

By repeated application of (4), $y A y$ can be reduced to $A^{\prime} y y$, where $A^{\prime}$ is the result of raising the subscript of each term in $A$ by 1 . Equation (3) then gives $A^{\prime} g_{0} y$, and repeated applications of (5) yield $g_{0} A y$, since $g_{0}$ lowers subscripts as it moves to the left, so long as it encounters no $g_{1}^{-1}$ 's (which it won't by the conditions in (5)) and so long as it has no $g_{0}^{-1}$ to the right of it (ditto). We can then reduce $g_{0} A y$ to $g_{0} B y$. Alternatively, we could have begun by reducing $y A y$ to $y B y$, and this then to $B^{\prime} y y, B^{\prime} g_{0} y$, and $g_{0} B y$. The resulting expressions are the same, completing the desired step in the verification of our normal-form assertion.

To determine $[S: G]_{\text {, }}$ and $[S: G]_{\ell}$, let us consider an element of $S$ whose expression in our reduced form is $A y$. Write $A$ as $B C^{\prime}$, where $B, C \in G$, and $C$ is chosen so that $C^{\prime}$ (as in the preceding paragraph) is the largest right-hand segment of $A$ not involving $g_{0}$ or $g_{0}^{-1}$. Then $B$ must either be empty or end in $g_{0}^{ \pm 1}$. We can thus, by (4) rewrite our element as $B y C$, and we observe that $B y$ will again be irreducible. It is easily deduced that every element of $S$ can be written uniquely either as a reduced expression for an element of $G$, or as $B y C$, where $B$ and $C$ are such reduced expressions, and $B$ either is empty, or ends in $g_{0}^{ \pm 1}$. It follows that $[S: G]_{2}=\boldsymbol{N}_{0}$, the cosets other than $G$ corresponding to these elements $B$, and that $G$ has right cancellation in $S$. On the other hand, since every element of $S$ can be written 
as $g$ or (nonuniquely) $g y$, we have $[S: G]_{\iota}=2$.

Larger values of $[S: G]_{2}$ could have been obtained by adjoining to $G$ still more free generators, $h_{\alpha, i}$ (where $i$ takes on all integer values, and $\alpha$ ranges over a large index set) and the relations $y h_{\alpha, i}^{ \pm 1}=h_{\alpha, i+1}^{ \pm 1} y$, for all $i$ and $\alpha$.

\section{REFERENCE}

1. P. M. Cohn, Universal Algebra, Harper and Row, 1965.

Received April 21, 1970. Part of this work was done while the author was supported by a Science Research Council fellowship.

Permanent affiliation:

HARVARD UNIVERSITY

BEDFORD COLLEGE, LONDON

University of CALIFornia, Berkeley 


\title{
PACIFIC JOURNAL OF MATHEMATICS
}

\author{
EDITORS
}

H. SAMELSON

Stanford University

Stanford, California 94305

C. R. Новву

University of Washington

Seattle, Washington 98105
J. DUGUNDJI

Department of Mathematics

University of Southern California

Los Angeles, California 90007

RICHARD ARENS

University of California

Los Angeles, California 90024

\section{ASSOCIATE EDITORS}

E. F. BECKENBACH

B. H. NeUManN

F. WOLF

K. YosHidA

\section{SUPPORTING INSTITUTIONS}

UNIVERSITY OF BRITISH COLUMBIA CALIFORNIA INSTITUTE OF TECHNOLOGY UNIVERSITY OF CALIFORNIA MONTANA STATE UNIVERSITY UNIVERSITY OF NEVADA NEW MEXICO STATE UNIVERSITY OREGON STATE UNIVERSITY UNIVERSITY OF OREGON OSAKA UNIVERSITY UNIVERSITY OF SOUTHERN CALIFORNIA
STANFORD UNIVERSITY UNIVERSITY OF TOKYO UNIVERSITY OF UTAH WASHINGTON STATE UNIVERSITY UNIVERSITY OF WASHINGTON

AMERICAN MATHEMATICAL SOCIETY CHEVRON RESEARCH CORPORATION TRW SYSTEMS

NAVAL WEAPONS CENTER 


\section{Pacific Journal of Mathematics}

\section{Vol. 36, No. $1 \quad$ November, 1971}

Norman Larrabee Alling, Analytic and harmonic obstruction on

nonorientable Klein surfaces ............................ 1

Shimshon A. Amitsur, Embeddings in matrix rings .............. 21

William Louis Armacost, The Frobenius reciprocity theorem and essentially bounded induced representations ....................... 31

Kenneth Paul Baclawski and Kenneth Kapp, Topisms and induced

non-associative systems ............................ 45

George M. Bergman, The index of a group in a semigroup ............ 55

Simeon M. Berman, Excursions above high levels for stationary Gaussian

processes....................................... 63

Peter Southcott Bullen, A criterion for $n$-convexity .............. 81

W. Homer Carlisle, III, Residual finiteness of finitely generated commutative

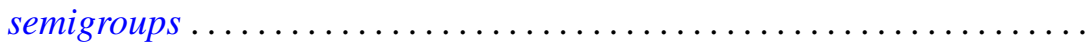

Roger Clement Crocker, On the sum of a prime and of two powers of

two ............................................ 103

David Eisenbud and Phillip Alan Griffith, The structure of serial rings . . . 109

Timothy V. Fossum, Characters and orthogonality in Frobenius

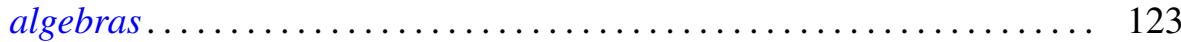

Hugh Gordon, Rings of functions determined by zero-sets . .......... 133

William Ray Hare, Jr. and John Willis Kenelly, Characterizations of Radon partitions...

Philip Hartman, On third order, nonlinear, singular boundary value

problems

David Michael Henry, Conditions for countable bases in spaces of

countable and point-countable type ..

James R. Holub, Hilbertian operators and reflexive tensor products ...

Robert P. Kaufman, Lacunary series and probability ..... . .

195

Erwin Kreyszig, On Bergman operators for partial differential equations in

two variables ................................

Chin-pi Lu, Local rings with noetherian filtrations . .

Louis Edward Narens, A nonstandard proof of the Jordan curve theorem...

S. P. Philipp, Victor Lenard Shapiro and William Hall Sills, The Abel summability of conjugate multiple Fourier-Stieltjes integrals. .

Joseph Earl Valentine and Stanley G. Wayment, Wilson angles in linear normed spaces

Hoyt D. Warner, Finite primes in simple algebras ...

Horst Günter Zimmer, An elementary proof of the Riemann hypothesis for an elliptic curve over a finite field... 